\title{
Context based motion and disparity vector prediction algorithm for H.264 based stereoscopic video coding
}

\author{
ABB Adikari ${ }^{\text {a)}}$, W.A.C. Fernando ${ }^{\text {b) }}$, and H. Kodikara Arachchic) \\ School of Engineering and Design, Brunel University, Uxbridge, UB8 3PH, UK \\ a)adikari.adika@brunel.ac.uk \\ b) anil.fernando@brunel.ac.uk \\ c)eesrhhk@brunel.ac.uk
}

\begin{abstract}
H.264 video coding standard supports 3D video through dedicated Stereo Video Information (SVI) Supplemental Enhancement Information (SEI) message. This SEI message allows main and auxiliary frames be coded into alternative pictures in which disparity information can also be utilized. However, due to the presence of both disparity and motion vectors at the neighborhood of a given macroblock, the H.264 predicted motion vector motion vector concept becomes less efficient. This paper presents a novel motion and disparity vector prediction algorithm that overcomes this drawback. Results show that the proposed algorithm achieves up to $1 \mathrm{~dB}$ gain compared to the conventional motion vector prediction algorithm.
\end{abstract}

Keywords: H.264, stereoscopic video, 3D video

Classification: Science and engineering for electronics

\section{References}

[1] K. Akiyama, N. Tetsutani, M. Ishibashi, S. Ichinose, and H. Yasuda, "Consideration on three-dimensional visual communication systems," IEEE J. Select. Areas Commun., vol. 9, no. 4, pp. 555-560, May 1991.

[2] A. Redert, E. Hendriks, and J. Biemond, "3-D scene reconstruction with viewpoint adaptation on stereo displays," IEEE Trans. Circuits Syst. Video Technol., vol. 10, no. 4, pp. 550-562, June 2000.

[3] J.-Y. Son and B. Javidi, "Three-dimensional imaging methods based on multiview images," J. Display Technol., vol. 1, no. 1, pp. 125-140, Sept. 2005.

[4] P. D. Gunatilake, M. W. Siegel, and A. G. Jordan "Compression of Stereo Video Streams," Proc. SPIE Stereoscopic Displays and Virtual Reality Systems, vol. 2177, San Jose, CA, pp. 258-268, Feb. 1994.

[5] S. Thanapirom, W. A. C. Fernando, and E. A. Edirisinghe, "A zerotree stereo video encoder," Proc. 2003 Int. Symp. Circuits and Systems, vol. 2, pp. 25-28, May 2003.

[6] ITU-T Recommendation H.264, "Advanced video coding for generic audiovisual," March 2005. 


\section{Introduction}

A glance into the past decade will verify that 3D visual communication technology [1] has received considerable interest as it intends to provide reality of vision. Various types of 3D capturing and displaying $[2,3]$ techniques have been developed in order to produce the same depth sensation as we experience in the real world. However, the accomplishment of 3D visual communication technology requires several other supporting technologies such as 3D image representation, handling, and compression for ultimate commercial exploitation. Many innovative studies on 3D visual communication technology were focused on developing efficient image compression algorithms [4, 5], which is one of the most significant fields in this evolution.

State-of-the-art video coding standard, H.264, provides a framework for 3D video coding through a Supplemental Enhancement Information (SEI) message called Stereo Video Information (SVI) message [6]. SVI informs the decoder that the coded video stream contains two views. Further it specifies whether they are arranged in field format or frame format and which of those views has been coded independently. Under field format, each view of a given time instance are coded into each field of interlaced video frame and under the frame format, alternate frames in the output order represents a view.

The disparity redundancies can also be exploited if each view is coded into separate frames. Under this configuration, there is a possibility that a given block in a picture from one view is coded with respect to a picture from the other view in the same time instance or in a previous time instance. However, in order to make the coded video compatible with traditional single view applications, it is necessary to code one of the two views independent of the other. This independently coded view is known as main view and it does not contain any disparity compensated blocks. In contrast, some of the blocks in a picture from the auxiliary stream may have been disparity compensated while the others are motion compensated.

H.264 has reduced the cost of coding motion vectors by coding the difference between the actual vector and a predicted vector. Therefore, the accuracy of the predicted motion vector is crucial to maximize the compression efficiency. This predicted vector is calculated based on vectors of surrounding blocks in the same slice, which have already been encoded [6]. However, the native H.264 prediction algorithm does not differentiate the disparity and the motion vectors which are highly unlikely to be correlated to each other. As a result, the predicted vector for a given macroblok in an auxiliary picture is unlikely to be accurate. Hence, the expected bit saving cannot be achieved. The proposed technique addresses this problem.

\section{Proposed Technique}

Similar to the native H.264 motion vector prediction algorithm, the proposed technique considers vectors $\mathrm{a}, \mathrm{b}, \mathrm{c}$ and $\mathrm{d}$, of the surrounding blocks $\mathrm{A}, \mathrm{B}, \mathrm{C}$ and D shown in the Fig. 1 to predict a vector for the current macroblock X. Some of these vectors may be motion compensated while the rest is disparity 


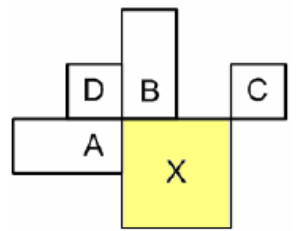

Fig. 1. Neighborhood used to predict a motion vector for the block X

compensated. Therefore, the first stage is to identify whether the current $\mathrm{MB}, \mathrm{X}$, is disparity compensated or motion compensated. If it is disparity compensated, only disparity compensated surrounding blocks are used for the proposed prediction and vise versa. Due to this division, there are five major cases to be treated in disparity vector prediction and five similar cases for the motion vector prediction. Table I describes these cases for disparity

Table I. Predicted disparity vector, xpred, for the block X

\begin{tabular}{|c|c|c|}
\hline \multirow[t]{3}{*}{1.} & \multicolumn{2}{|c|}{ Only one of $A, B$ and $C$ are disparity compensated } \\
\hline & Native & $\mathbf{x}_{\text {pred }}=$ select_disparity_vector $(\mathbf{a}, \mathbf{b}, \mathbf{c})$ \\
\hline & Proposed & Uses the native algorithm \\
\hline \multirow[t]{3}{*}{2} & \multicolumn{2}{|c|}{ Only two of $A, B$ and $C$ are disparity compensated } \\
\hline & Native & $\mathbf{x}_{\text {pred }}=\operatorname{median}(\mathbf{a}, \mathbf{b}, \mathbf{c})$ \\
\hline & Proposed & $\begin{array}{l}\left(\mathbf{t}_{1}, \mathbf{t}_{2}\right)=\text { select_disparity_vectors }(\mathbf{a}, \mathbf{b}, \mathbf{c}) \\
\text { if D is disparity compensated } \\
\mathbf{x}_{\text {pred }}=\operatorname{median}\left(\mathbf{t}_{1}, \mathbf{t}_{2}, \mathbf{d}\right) \\
\text { else } \\
\qquad \mathbf{x}_{\text {pred }}=\text { average }\left(\mathbf{t}_{1}, \mathbf{t}_{2}\right)\end{array}$ \\
\hline \multirow[t]{3}{*}{3} & \multicolumn{2}{|c|}{ All $A, B$ and $C$ are disparity compensated } \\
\hline & Native & $\mathbf{x}_{\text {pred }}=\operatorname{median}(\mathbf{a}, \mathbf{b}, \mathbf{c})$ \\
\hline & Proposed & Uses the native algorithm \\
\hline \multirow[t]{3}{*}{4} & \multicolumn{2}{|c|}{ Only $D$ is disparity compensated } \\
\hline & Native & $\mathbf{x}_{\text {pred }}=\operatorname{median}(\mathbf{a}, \mathbf{b}, \mathbf{c})$ \\
\hline & Proposed & $\mathbf{x}_{\text {pred }}=\mathrm{d}$ \\
\hline \multirow[t]{3}{*}{5} & \multicolumn{2}{|c|}{ All A, B, C and D are motion compensated } \\
\hline & Native & $\mathbf{x}_{\text {pred }}=\operatorname{median}(\mathbf{a}, \mathbf{b}, \mathbf{c})$ \\
\hline & Proposed & $\mathbf{x}_{\text {pred }}=0$ \\
\hline
\end{tabular}


vector prediction and corresponding predicted vector, $\mathrm{x}_{\mathrm{pred}}$, when the native H.264 algorithm and the proposed algorithm are applied.

Even though the native algorithm produces an acceptable prediction for the first and the third cases, modifications are necessary for the second, the fourth and the fifth cases. For the second case, the median of $\mathrm{d}$ and two available disparity vectors out of $\mathrm{a}, \mathrm{b}$, and $\mathrm{c}$ is used, if $\mathrm{d}$ is also disparity compensated. Otherwise, the average of the two disparity vectors is used. In the fourth case, since $d$ is the only disparity vector, it is reasonable to consider $\mathrm{d}$ as the predicted vector. For the final case however, there is no simple disparity vector prediction technique since all the neighboring blocks have been motion compensated. Therefore, the predicted motion vector is set to zero. Similar technique is applied in treating corresponding motion vector prediction cases as well.

\section{Results}

The simulation results were obtained for 'Crowd' and 'Booksale' stereoscopic sequences. Picture dimensions of the both sequences are same at $640 \times 240$. The rate control is disabled during this simulation and various quantizer parameters are used. Figure 2 shows the 'PSNR vs. bits per pixel' graph for the luminance ( $\mathrm{Y}$ ) signal for 90 frames of the auxiliary stream (right stream) of the 'Crowd' sequence whereas Fig. 3 shows the same for the 'Booksale' sequence. These graphs clearly show that the proposed technique can yield a gain of up to $1 \mathrm{~dB}$ compared to the native H.264 prediction algorithm.

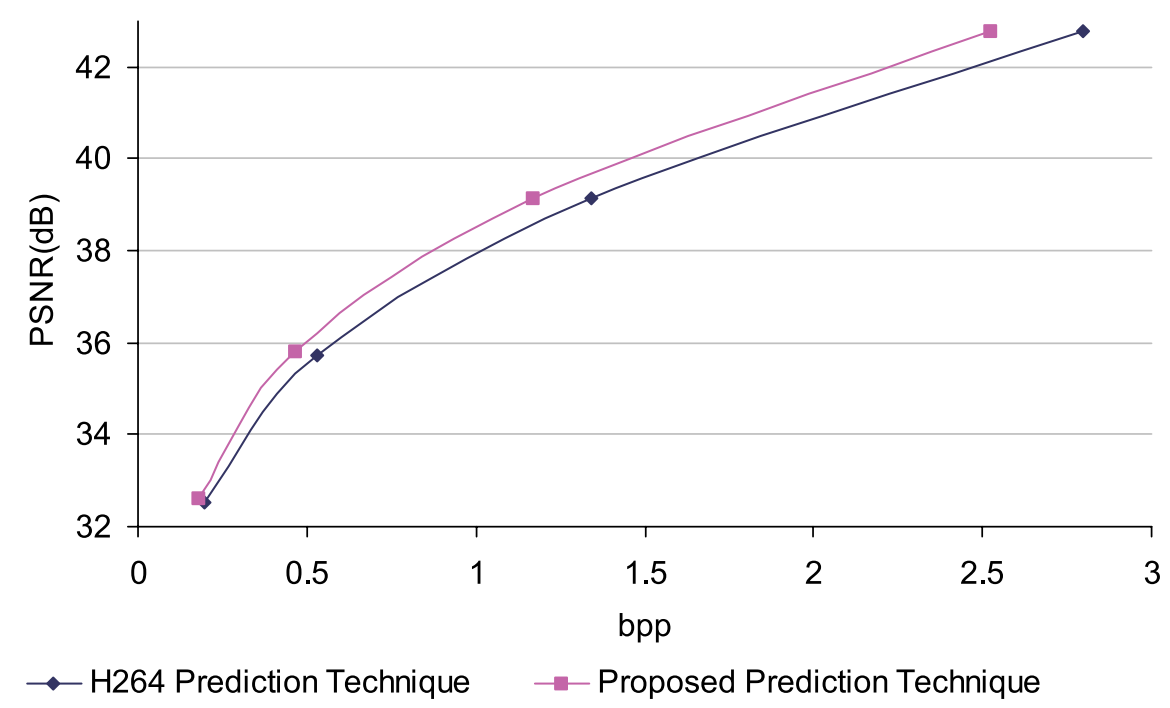

Fig. 2. Performance comparison of native prediction algorithm with the proposed prediction algorithm the 'Crowed' Sequence 


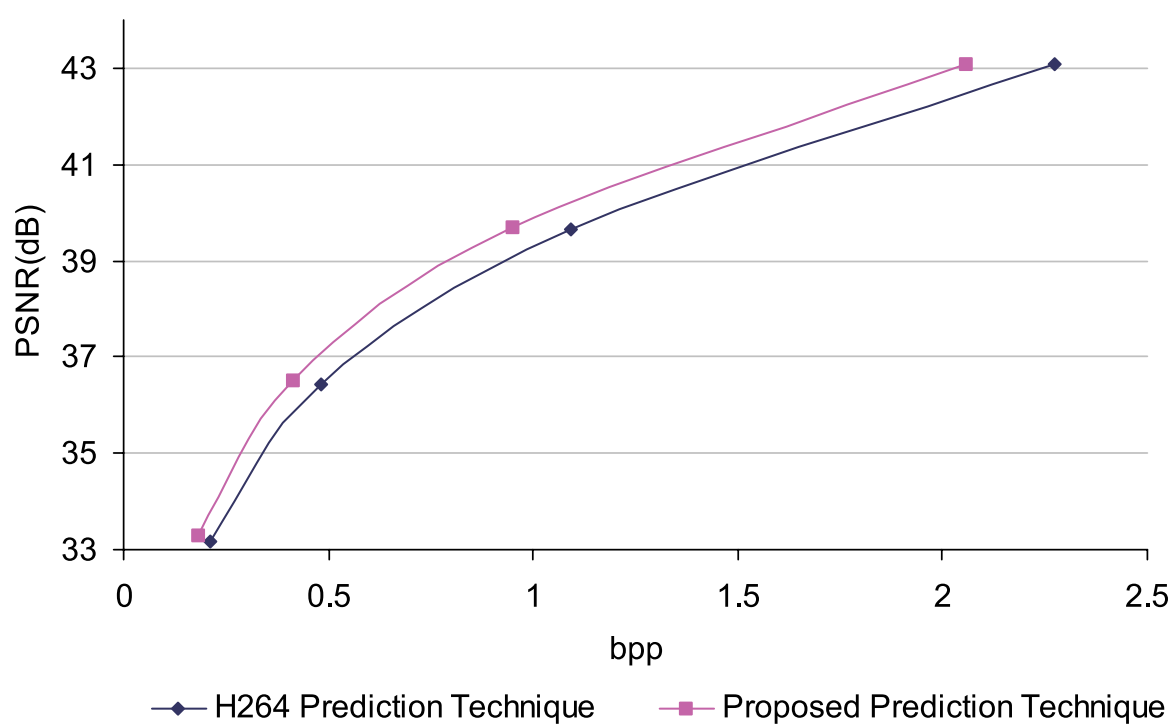

Fig. 3. Performance comparison of native prediction algorithm with the proposed prediction algorithm the 'Book Sale' Sequence

\section{Conclusions}

In this paper a novel for motion and disparity vector prediction algorithm for H.264 based stereoscopic video codec is proposed. The experimental results show that proposed algorithm improves the objective quality up to $1 \mathrm{~dB}$ compared to the native H.264 at the same bit rate.

\section{Acknowledgments}

Authors would like to thank EPSRC for funding this research project. 